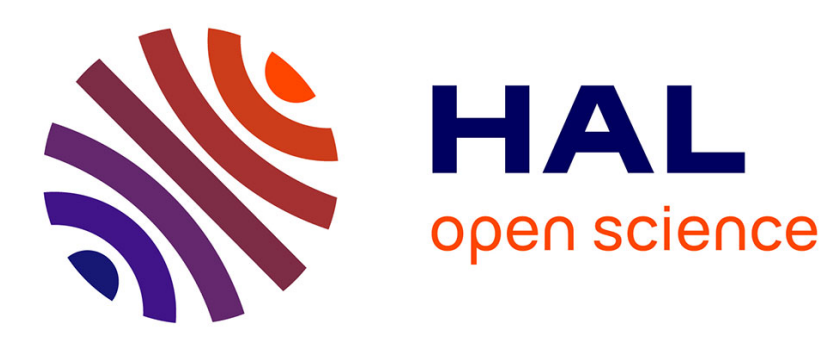

\title{
Transitions professionnelles et transactions identitaires : expériences, épreuves, ouvertures \\ Thérèse Perez-Roux
}

\section{To cite this version:}

Thérèse Perez-Roux. Transitions professionnelles et transactions identitaires : expériences, épreuves, ouvertures. Pensée Plurielle - Parole, pratiques et réflexions du social , 2016, 41 (1), pp.81-93. 10.3917/pp.041.0081 . hal-01716256

\section{HAL Id: hal-01716256 \\ https://hal.science/hal-01716256}

Submitted on 27 Feb 2018

HAL is a multi-disciplinary open access archive for the deposit and dissemination of scientific research documents, whether they are published or not. The documents may come from teaching and research institutions in France or abroad, or from public or private research centers.
L'archive ouverte pluridisciplinaire HAL, est destinée au dépôt et à la diffusion de documents scientifiques de niveau recherche, publiés ou non, émanant des établissements d'enseignement et de recherche français ou étrangers, des laboratoires publics ou privés. 
Perez-Roux, T. (2016). Transitions professionnelles et transactions identitaires : expériences, épreuves, ouvertures. Revue Pensée plurielle, $\mathrm{n}^{\circ} 41,81-93$.

\title{
Transitions professionnelles et transactions identitaires : expériences, épreuves, ouvertures
}

Thérèse Perez-Roux ${ }^{1}$, Laboratoire Interdisciplinaire de Recherches en Didactique Education et Formation (LIRDEF EA-3749), Montpellier 3.

Résumé

Dans un contexte de transformation du marché du travail, les transitions professionnelles se diversifient. La contribution s'intéresse aux effets de ces transitions sur les dynamiques identitaires des acteurs. Sur la base des résultats de deux études conduites auprès de professionnels qui se réorientent dans un nouveau métier ou font la démarche d'un retour en formation, plusieurs éléments sont mis en lumière : l'expérience de la transition est analysée à la fois comme un moment de perte, une épreuve plurielle et un espace de développement potentiel dans lequel de puissants enjeux identitaires sont à l'œuvre.

Mots clés : transitions professionnelles, identité professionnelle, expérience, épreuves, formation

\section{Professional transitions and identity transactions: experiences, hardships, openings}

\begin{abstract}
In a context of evolution world of work, professional transitions diversify. The contribution focuses on the effects of these transitions on actor identity dynamics. Based on the results of two studies conducted with professionals who turn into a new work or make the process of returning to training, several elements are highlighted : the experience of transition is analyzed as both a time of loss, a plural hardship and a potential development space in which identity stakes are discussed.
\end{abstract}

Keywords

Professional transitions, professional identity, experience, hardships, training

\section{Introduction}

Dans un contexte de transformation du marché du travail, la question des transitions se pose de façon significative; elle s'inscrit dans des trajectoires professionnelles liées à la fois aux contextes socio-économiques et culturels mais aussi aux stratégies singulières des acteurs (Perez-Roux et Balleux, 2014). En effet, les travailleurs sont de plus en plus amenés à s'adapter, à développer d'autres compétences, à bifurquer vers des secteurs plus «porteurs » au plan économique, plus valorisants au plan professionnel et/ou personnel, etc. Par ailleurs, comme l'évoque Martuccelli (2014) la transition renvoie à "l'expérience de la modernité » (p.11) avec ses turning points et ses bifurcations, qui attestent, pour les individus, de la

\footnotetext{
${ }^{1}$ Professeure des universités, Département des Sciences de l'Education, UFR Education et Sciences, Université P. Valéry, Montpellier 3
} 
Perez-Roux, T. (2016). Transitions professionnelles et transactions identitaires : expériences, épreuves, ouvertures. Revue Pensée plurielle, $n^{\circ} 41,81-93$.

volonté « d'avoir plusieurs vies en une seule et de disposer de dispositifs de réversibilité dans leur parcours de vie » (Mazade et Hinault, $2014: 3$ ).

Cette contribution s'intéresse à des formes de transitions « désirées » dans le sens où ce sont les acteurs eux-mêmes qui ont décidé de s'engager dans un processus de transformation ; bien que ce projet leur semble globalement réalisable, il constitue le plus souvent un véritable défi. Deux situations de transition seront ici étudiées : la première concerne des réorientations professionnelles vers un nouveau métier; la seconde interroge le retour en formation de professionnels expérimentés. Entre phénomènes de rationalisation des choix et enjeux identitaires cachés, entre logiques d'acteurs et effets de contexte, de nombreux mobiles coexistent et s'agrègent, organisés de façon plus ou moins cohérente autour d'un projet porté par le sujet. Le changement impulsé volontairement est donc toujours situé dans le temps, l'espace, l'organisation de travail et ses collectifs. Il est plus ou moins soutenu par l'institution de rattachement (perspectives de promotion, valorisation, financements, aménagements des conditions de travail, etc.). Enfin, il invite -et s'invite dans- la sphère du personnel qui va être affectée par le mouvement d'ensemble dans lequel s'inscrit le sujet en transition.

\section{Une approche conceptuelle intégrée}

Notre approche des transitions professionnelles repose sur un certain nombre de concepts articulés, présentés dans les trois sous-parties suivantes.

\section{A. La transition : un entre deux orienté vers/par un projet}

Tout au long du cycle de vie, les périodes de transition sont nombreuses et diversifiées. Plus spécifiquement, dans la sphère professionnelle, celles-ci sont associées à des moments différents : passage de l'école ou de la formation à l'emploi (Rose, 2009), phases d'insertionsocialisation dans de nouveaux contextes de travail (Lanéelle et Perez-Roux, 2014), périodes de réorientation professionnelle désirées ou subies (perte d'emploi, transformation des prescriptions), etc. Ainsi, la transition peut être définie comme : « un espace / temps de passage inscrit au cœur d'un changement, assumé ou non, abouti ou non, et qui nécessite de l'individu la mise en œuvre de stratégies d'adaptation pour mieux gérer éléments de rupture et (re)construction de continuités » (Balleux \& Perez-Roux, 2013 : 102). Il s'agit aussi de gérer les tensions entre (re)positionnement personnel au regard de compétences revendiquées/attendues/à construire et nouveaux espaces relationnels qui intègrent enjeux de place et jeux de pouvoir. De fait, le mouvement de passage que constitue la transition réactive des éléments existentiels et professionnels puissants.

Pour Dupuy et Le Blanc (2001), l'individu utilise la transition pour faire des choix dans un environnement qui se transforme. Saisissant contraintes et opportunités du contexte, il décide, s'engage, se met en projet. La transition est alors envisagée comme une chance de développement de la carrière ou comme moyen de renforcer/ retrouver ses valeurs, comme une " phase de reconstruction active des valeurs et des normes fondant la reconnaissance et la valorisation de soi et autrui »(Dupuy \& Le Blanc, 2001 : 68). En ce sens, les stratégies développées au cours des périodes de transition intègrent aussi des formes de socialisation plurielles et actives qui participent à la résolution d'un certain nombre de tensions inter et intra-psychiques liées au changement. Au-delà des nouvelles transactions relationnelles 
Perez-Roux, T. (2016). Transitions professionnelles et transactions identitaires : expériences, épreuves, ouvertures. Revue Pensée plurielle, $n^{\circ} 41,81-93$.

susceptibles de s'opérer durant la transition, la mise en projet de soi vient réinterroger certains acquis et brouiller les repères antérieurs. Les acteurs mettent en œuvre des stratégies multiples et parfois inconscientes (Crozier \& Friedberg, 1977) pour reconstruire du sens dans une relation individu / environnement transformée. Ils se saisissent des opportunités, réactivent des potentialités laissées en sommeil, se projettent dans l'avenir. Cette dynamique est traversée par un certain nombre de tensions, vécues parfois comme autant d'épreuves qu'il faut parvenir à surmonter.

\section{B. L'expérience de la transition comme (sou)mise à l'épreuve}

Si la notion d'expérience renvoie en partie au contrôle ajusté des situations de travail (stratégies, routines, résolutions de problèmes typiques, savoir-faire et savoir y faire), elle concerne aussi l'intensité et la signification d'une situation vécue par un acteur, de façon positive ou négative. Dans ce cas, l'expérience ne s'inscrit pas dans un processus fondé sur la répétition des situations mais elle est décisive dans le sens où elle affecte en profondeur le rapport au travail. Il s'agit en quelque sorte d'une "expérience identitaire qui n'est pas de l'ordre du savoir théorique ou pratique mais du vécu et où se mêlent intimement des aspects personnels et professionnels : sentiment de maîtrise, découverte de soi dans le travail, etc. » (Tardif et Lessard, 1999 : 40). Elle renvoie au vécu de l'activité qui à la fois transforme le sujet et modifie son rapport au monde. Dans l'espace/temps de la transition, cette expérience est soumise à un système de tensions constitutif d'un certain nombre d'épreuves. Boltanski \& Chiapello (1999) définissent ces épreuves comme une épreuve de force, un «événement au cours duquel des êtres, en se mesurant, révèlent ce dont ils sont capables et même, plus profondément, ce dont ils sont faits » (p. 74). L'épreuve prend donc au sérieux la dynamique ouverte de l'expérience, ce qui génère inévitablement de l'incertitude. Dans une perspective intégrant la singularité des situations, Martuccelli (2006) définit les épreuves comme des défis plus ou moins partagés, inscrits dans une histoire culturelle (du métier par exemple) et construits au plan social. C'est avec leurs propres ressources que les individus sont contraints d'affronter ces défis qui sont vécus de manière intime, subjective, existentielle. En même temps, ces défis font sens/sont repérables pour un ensemble d'acteurs et renvoient, de fait, à une dimension sociale. Cela suppose de traduire de manière intelligible l'expérience des «transitants » et de rendre compte de leurs vécus singuliers, qui viennent enrichir des rapports de sens partagés. Ces épreuves sont constituées d'un faisceau d'éléments enchevêtrés. Les prendre en considération amène à interroger ce qui se joue, se noue et de dénoue au plan identitaire.

\section{Les transactions identitaires : un éclairage sur la dynamique des transitions}

Prenant appui sur un certain nombre d'apports de la psychologie sociale qui soulignent le caractère hétérogène des situations de transition (Dupuy \& Le Blanc, 2001; Mègemont \& Baubion-Broye, 2001), l'approche des dynamiques identitaires choisie dans cette contribution s'ancre plus résolument dans une perspective sociologique. Dans le prolongement des travaux de Dubar (1992, 2000) et de Kaddouri (2006), le modèle d'intelligibilité des dynamiques identitaires est organisé à partir d'une «triple transaction biographique-relationnelle- intégrative »(Perez-Roux, 2011). La première (biographique) intègre les tensions entre continuité et changement; la seconde (relationnelle), celles du 
Perez-Roux, T. (2016). Transitions professionnelles et transactions identitaires : expériences, épreuves, ouvertures. Revue Pensée plurielle, $n^{\circ} 41,81-93$.

rapport de soi à des autrui pluriels ; la troisième (intégrative) tente de combiner cohérence du moi (ou sentiment d'unité) et diversité des registres de pensée et d'action.

Tout d'abord, l'axe continuité / changement est appréhendé à travers le parcours professionnel durant lequel l'individu doit nécessairement s'adapter. Un couplage s'opère alors entre histoire du sujet et modifications plus ou moins souhaitées et/ou contrôlées dans le contexte professionnel. Dans quelle mesure la transition s'inscrit dans une continuité et prend-elle en compte des éléments de rupture (réels ou symboliques) ? Comment permet-elle de s'ancrer dans le passé tout en se projetant dans un avenir qui sera différent et qui risque de transformer, de faire perdre d'anciens repères ?

Cette dynamique temporelle se combine avec une dimension relationnelle. Chacun élabore une image de soi en relation - accord, tension, contradiction - avec celles attribuées par autrui. Ce rapport de soi à autrui, engageant des phénomènes de reconnaissance ou de non reconnaissance se révèle essentiel pour la construction de l'identité professionnelle. Quelles formes de légitimité pour soi-même et pour les autres peuvent se (re)construire et quel sentiment de reconnaissance d'autrui peut émerger dans un contexte différent ?

Enfin, la troisième transaction mobilisée pour comprendre les remaniements identitaires (transaction intégrative) s'inscrit dans une approche psychosociale. Elle prend en compte la tension entre unité / cohérence de l'individu fortement portée par des systèmes de valeurs (souvent revendiquées comme organisatrices des choix) et par la diversité des facettes et des registres d'action mobilisés par ce même individu dans les sphères sociale, professionnelle et privée. Les individus en période de transition parviennent-ils à investir de nouveaux espaces, à développer de nouvelles compétences, à mobiliser ou remobiliser des potentialités laissées en sommeil pour répondre aux attentes de l'organisation/de l'institution ? Quelle cohérence pour soi-même et pour autrui dans l'approche d'un nouveau métier, dans la perspective d'une nouvelle fonction, dans le passage d'une identité professionnelle affirmée à une identité brouillée?

Cet ensemble de questions vient nourrir la réflexion sur : a) les transitions et les épreuves traversées ; b) les remaniements identitaires que ces transitions sont susceptibles de générer.

\section{Terrains investigués et choix méthodologiques}

Entre 2008 et 2015, nous avons conduit plusieurs recherches pour comprendre « ce qui se joue » lorsque les acteurs se trouvent dans des espaces/temps de transition. Documenter les transitions professionnelles suppose : a) de s'inscrire dans la durée et de prendre au sérieux les retours sur le parcours, l'inscription dans le présent et la projection dans le futur ; b) de saisir la qualité des relations humaines, les éléments de congruence ou de discordance entre définition de soi comme professionnel et regards d'autrui ; c) de comprendre le type de cohérence entre registres de pensée et d'action, entre rôles à tenir et places revendiquées dans l'espace personnel, social et professionnel. Ainsi cette approche donne des clés de lecture sur les formes de remaniements identitaires. Dans ces recherches un questionnaire exploratoire initie le recueil de données. Sur la base de questions fermées, de questions à choix multiples, de questions ouvertes, l'enquête permet de repérer : a) les caractéristiques sociologiques (sexe, âge, statut, ancienneté, parcours, etc.) ; b) les représentations et valeurs, les formes d'implication choisies, le rapport à la pratique professionnelle et l'inscription dans les contextes de travail. Au regard de ces premiers résultats, des sujets volontaires sont retenus pour réaliser un entretien semi directif qui invite à approfondir, à préciser, à contextualiser 
Perez-Roux, T. (2016). Transitions professionnelles et transactions identitaires : expériences, épreuves, ouvertures. Revue Pensée plurielle, $n^{\circ} 41,81-93$.

telle ou telle réponse et à développer des aspects moins stabilisés, parfois même des éléments de l'expérience jusque-là peu conscientisés.

Deux enquêtes sont ici mobilisées pour rendre compte des transactions à l'œuvre dans les moments de transition.

La première a été conduite en 2008 auprès d'anciens professionnels du transport au moment où ils décident de devenir enseignants $(n=15)^{2}$. Après la réussite d'un concours de recrutement ${ }^{3}$ de l'Education Nationale, ils suivent une année de formation professionnalisante (alternance de trois semaines dans un lycée professionnel au titre d'enseignant-stagiaire et une semaine en centre de formation). Cette bifurcation (seconde carrière) vers l'enseignement technique peut être considérée comme une «transition désirée » qui révèle un certain nombre de problèmes liés au passage d'un univers professionnel à un autre, très différent. Les analyses présentées dans les parties suivantes mobilisent une partie des résultats de cette étude, accessibles en ligne (Perez-Roux, 2010, 2011).

La seconde enquête a été conduite entre 2013 et 2015 auprès de professionnels travaillant dans différents secteurs (éducation, insertion ou formation) et en reprise d'étude pour obtenir un niveau master $(n=17)^{4}$. Ce retour en formation est sous tendu par deux visées essentielles : confirmer, approfondir, valoriser une fonction dans des métiers «adressés à autrui » ou se réorienter vers la formation des adultes (Perez-Roux, à paraître).

Les trois parties qui suivent présentent une méta-analyse sur la base de ces recherches dont les résultats donnent à comprendre l'expérience de la transition comme à la fois un moment de perte, une épreuve plurielle et un espace de développement potentiel dans lequel de puissants enjeux identitaires sont à l'œuvre.

\section{L'expérience de la transition comme « espace-temps de perte »}

A quelques exceptions près, les professionnels suivis dans les deux études étaient/sont reconnus pour leur travail et impliqués dans leur organisation. Cette implication professionnelle peut être analysée à partir du tryptique «sens-repères-contrôle » décrit par Mias (1998). Pour l'auteure, le sens renvoie à la signification que l'on attribue à ses actions ; il relève de l'intentionnalité et donne une cohérence à l'ensemble des éléments d'une situation. Les repères ont une fonction constructive et équilibrante dans la mesure où ils vont permettre à la fois : a) d'orienter les conduites en fonction d'un passé commun et de valeurs partagées dans un groupe identifié ; b) de s'ouvrir à un espace de réflexion par des re-négociations à l'échelle interindividuelle, collective et/ou organisationnelle ; c) d'engager des actions durables et profondes soumises à l'approbation et au soutien des autres. Le contrôle, enfin, correspond à la possibilité de se sentir responsable et libre de ses choix, ce qui suppose la

\footnotetext{
2 Un premier recueil de données par questionnaire a été réalisé auprès de 61 futurs enseignants de l'enseignement professionnel parmi lesquels 15 futurs professeurs de conduite routière. Des entretiens approfondis ont ensuite été conduits avec cinq de ces professionnels : le premier en janvier, le second en juin 2008.

${ }^{3}$ Concours de recrutement de Professeur de Lycée Professionnel

${ }^{4}$ Un premier recueil de données prend appui sur la mise en récit écrite de parcours professionnels, dans une dimension réflexive, invitant à croiser expérience et savoirs de la formation. Le corpus $(\mathrm{n}=17)$ a été analysé à partir du logiciel Alceste. De façon complémentaire, un deuxième recueil de données a été réalisé à partir d'entretiens semi-directifs conduits quelques mois après la sortie de formation, auprès de 8 personnes volontaires.
} 
Perez-Roux, T. (2016). Transitions professionnelles et transactions identitaires : expériences, épreuves, ouvertures. Revue Pensée plurielle, $n^{\circ} 41,81-93$.

construction par le sujet de repères fiables à partir desquels il peut s'orienter et orienter son action.

Si chaque élément du triptyque «sens-repère-contrôle » possède sa propre autonomie, leur combinaison est « fondamentale dans la structure de l'implication professionnelle et essentielle pour la compréhension des conduites adoptées par les acteurs professionnels » (Mias, 1998, p. 99). En effet, chacun semble donner du sens à son action si celle-ci entre, au moins en partie, en cohérence avec des représentations et des valeurs mobilisées tout au long de sa trajectoire professionnelle antérieure. Par ailleurs, l'implication reste liée aux repères pris dans différents espaces, traversés avec des statuts différents. Enfin, se pose la question du contrôle de la situation et des marges de manœuvre, réelles ou supposées par les acteurs, dans le contexte de travail.

Les professionnels interrogés font état de nombreux brouillages dans les repères antérieurs et d'une expérience de la transition relativement complexe à gérer.

Les futurs enseignants de conduite routière (enquête 1) passent d'un espace professionnel bien identifié (salarié d'une entreprise de transport, moniteur d'auto-école, formateurs d'adultes dans le secteur de la conduite routière) avec fiche de poste, règles de fonctionnement, partage de certaines valeurs, positionnement social, etc. à un espace brouillé, celui de l'Education Nationale avec ses hiérarchies internes, ses lourdeurs administratives, ses règles implicites. Les professionnels se disent surpris par les logiques de leurs collègues des autres disciplines (voire de la leur), ou par les attitudes de leurs élèves. Du point de vue de l'établissement d'affectation, ils se sentent parfois relégués dans une discipline peu valorisée, alors que par ailleurs, devenir enseignant était plutôt vécu comme une forme d'ascension sociale. Les entretiens sont révélateurs d'un déficit de repères à la fois institutionnels, organisationnels et humains et d'un manque de contrôle de la situation d'enseignement. En effet, enseigner dans leur domaine suppose d'assurer quatre types de contenus (maintenance, transport, logistique, législation) diversement appréciées par les élèves. Rien ne va de soi et l'expérience passée dans le transport routier ou dans l'accompagnement de jeunes et d'adultes, bien que mobilisatrice, ne suffit pas à faire entrer les élèves dans les apprentissages scolaires.

La complexité de cette situation est accrue par le statut de stagiaire en formation. L'accompagnement sur le terrain du stage peut être assuré par un enseignant plus jeune et plus expérimenté, face auquel le «débutant» craint de perdre la face quand la situation pédagogique échappe ou lorsque les pratiques sont réinterrogées (évaluées) au regard des attendus du nouveau métier (référentiel de compétences). De plus, comme l'évoque ce stagiaire, l'alternance elle-même conduit à un positionnement délicat : " on se sent chez nous ni dans l'établissement ni à l'IUFM, nulle part!» (Patrick, 45 ans, futur professeur en conduite routière). Cette situation d'entre-deux crée des discontinuités qui nuisent au suivi des élèves, empêchant de se poser, selon Patrick, comme enseignant référent : « du coup, pour les élèves, je ne suis pas le seul maître à bord dans ma classe ».

Pour le second groupe de professionnels en reprise d'études, le retour en formation s'inscrit à la fois dans une dynamique de progrès et génère son lot d'incertitudes, de craintes vis-à-vis du niveau d'exigence et des capacités à y répondre. La force de l'expérience leur apparaît souvent bien décalée vis-à-vis des contenus théoriques et méthodologiques proposés dans le master.

L'expérience de la transition peut ainsi générer, sur un temps au moins, une véritable crise de sens : construire de nouveaux repères, requestionner ses valeurs, se mettre en danger en expérimentant des procédures inhabituelles, se retrouver en fragilité sur tel ou tel domaine 
Perez-Roux, T. (2016). Transitions professionnelles et transactions identitaires : expériences, épreuves, ouvertures. Revue Pensée plurielle, $n^{\circ} 41,81-93$.

alors qu'auparavant, on était reconnu comme un adulte autonome et responsable, comme un professionnel aguerri. La transition opère alors comme un espace-temps de dés-implication provisoire, avec le sentiment : a) d'avoir perdu, au moins en partie, ses repères antérieurs ; b) d'avoir peu de marges de manœuvre pour penser et agir de manière efficiente dans le nouvel espace de travail ou de formation; c) de se perdre dans l'univers de savoirs théoriques nouveaux, denses, opaques, vécus parfois comme disjoints de la pratique. L’intégration de ces savoirs pluriels à son propre système de référence demande du temps.

\section{La transition et ses épreuves plurielles}

A l'appui de ces enquêtes, trois épreuves communes semblent traverser l'espace-temps de la transition: l'épreuve de l'altérité, l'épreuve des savoirs et l'épreuve de soi. Bien que coprésentes, elles s'expriment différemment selon les contextes mais aussi selon les individus, leur parcours, leurs aspirations et leur plus ou moins grande sérénité/adaptabilité face au changement.

\section{A. L'épreuve de l'altérité}

La transition d'un métier vers un autre ou le retour en formation renvoient inévitablement à la question de l'altérité, dont une des dimensions concerne les rapports à autrui : oscillant entre conflit et partage, ces rapports peuvent susciter des questionnements, des malentendus, du désir (Briançon, 2012). La transition vers le métier enseignant, où l'interaction humaine est au cœur des processus d'enseignement/apprentissage, interpelle l'altérité à différents niveaux : celui des élèves qui tantôt entrent dans les propositions de l'enseignant, tantôt se désintéressent voire gênent la mise en place de situations pédagogiques conçues en amont. L'autre est alors perçu comme celui qui empêche la réalisation du travail tel que le professionnel souhaiterait le faire ; il tient une position différente, résiste, là où l'on comptait sur son adhésion, s'inscrit dans une logique différente (Cifali, 1999). Ces écarts rappellent les enjeux de la transmission des savoirs et de leur appropriation, de l'efficience de l'enseignant et, plus largement de son utilité sociale. Le passage dans un nouvel univers professionnel suppose aussi la rencontre avec les pairs. Dans la transition, l'espace de travail ou de formation génère des formes relationnelles diverses : processus d'intégration au sein du (des) groupe(s) et de différenciation, alliances partielles, conflits d'intérêts, choix de pratiques viennent brouiller les ancrages relationnels préalablement construits et les rapports de place. L'autre est ici, potentiellement, celui qui bouscule, remet en cause le travail dans un espace/temps où les anciens repères professionnels sont fragilisés. L'altérité renvoie enfin à l'organisation et ses logiques parfois incompréhensibles, tant au niveau de l'institution qui forme $^{5}$ que de celle qui évalue ${ }^{6}$ l'ancien professionnel redevenu apprenant. Ainsi, les personnes en transition expriment un positionnement délicat par rapport au groupe, aux formateurs, aux attentes de la formation : «au niveau des enjeux... sans parler même de réorientation, c'est compliqué... Aujourd'hui j'ai 30 ans d'expérience, je viens me confronter à des petits jeunes qui ont 20 ans de moins que moi. Qu'est-ce que vaut mon expérience ? Est-

\footnotetext{
${ }^{5}$ Institut Universitaire de Formation des Maîtres pour le groupe 1 ; Université pour le groupe 2

${ }^{6}$ Employeur Education Nationale pour le groupe 1 ; enseignants du master pour le groupe 2.
} 
Perez-Roux, T. (2016). Transitions professionnelles et transactions identitaires : expériences, épreuves, ouvertures. Revue Pensée plurielle, $n^{\circ} 41,81-93$.

ce que j'ai le même discours que les étudiants ou que des jeunes comme moi qui sont insérés depuis pas trop longtemps dans le milieu professionnel ? Déjà pour moi c'était important mais pour quelqu'un qui a lâché les études depuis 20 ou 30 ans, c'est hyper important, c'est : quelle valeur j'ai aujourd'hui ? » (Marc, 33 ans, en réorientation, formation Master 2).

Derrière cette épreuve de l'altérité se jouent des questions de reconnaissance, activées dans le temps de la transition. En effet, le passage par la formation pour se réorienter, développer de nouvelles compétences ou les confirmer, brouille parfois les repères, oblige à changer le point de vue. La légitimité n'est plus si évidente : il faut prouver et se prouver que l'on peut désormais entrer dans un nouvel espace de travail/de formation et se sentir soutenu, accepté, valable. Cette épreuve de la reconnaissance est souvent traduite dans l'après-coup: « personnellement, je n'ai pas le sentiment d'avoir changé de positionnement mais je mesure que pour certains de mes collègues et pour ma hiérarchie j'ai gagné en crédibilité et en reconnaissance » (Claire, 52 ans, cadre de santé, en formation Master 2).

\section{B. L'épreuve des savoirs}

Dans les deux formations professionnelles que nous avons étudiées, les dispositifs proposés invitent le formé à une prise de recul sur ses pratiques et à une explicitation du fondement de ses actes. Le praticien adopterait ainsi une posture d'extériorité en mettant à distance sa situation de travail, en se donnant la possibilité de la questionner, de l'analyser, de l'alimenter, en clarifiant d'une part ses propres savoirs implicites et en s'adossant, d'autre part, sur des savoirs constitués. Cette perspective ne va pas de soi. Le temps de la transition, combinée avec l'espace de la formation nécessite d'articuler différemment savoirs formalisés, savoirs formalisables et savoirs tacites (Alsène, Gamache et Lejeune, 2002). Pour les futurs enseignants de conduite routière, il s'agit de prendre appui sur des savoirs tacites ou expérientiels qu'il faut à présent formaliser et organiser (pôle didactique), puis faire vivre en situation de classe (pôle pédagogique). Réussir leur transformation en savoirs formalisables s'effectue aussi par le jeu des interactions dans le groupe de formation : questionnement, controverses professionnelles, accords sur tel ou tel point, présentations de plans de cours, analyse a posteriori de l'activité enseignante. L'action du collectif des formés et surtout des formateurs consiste ensuite à faire advenir des savoirs formalisés, transférables à d'autres situations de travail et de formation.

Pour les professionnels retournant à l'université, le rapport aux savoirs théoriques et méthodologiques reste délicat. Ces derniers ont déjà construit des compétences professionnelles dans leur milieu professionnel d'origine et le retour en formation suppose le développement d'autres registres de pensée et d'action. Si les compétences attendues dans le cadre du travail renvoient au pouvoir d'agir en situation, celles de la formation intègrent savoir dire, savoir questionner, savoir se distancier, tout en cherchant les modes d'articulation pratique-théorie-pratique les plus pertinents.

L'épreuve consiste ici à accepter le changement de focale, à requestionner les allant-de-soi, à assumer les moments de relative opacité des contenus proposés. Le travail d'appropriation est présenté comme couteux, déstabilisant, et en même temps très stimulant comme le décrit cette professionnelle inscrite en master : « il y a eu la présentation des matières, des enseignants. Là, j'ai pris la mesure de ce qu'il y avait à faire et j'ai pas eu le temps de me retourner... puis le contenu m'a plu, j'ai trouvé ça riche, j'ai complètement adhéré. Je me sentais portée ! » (Rosine, 41 ans, formatrice/accompagnatrice VAE, en formation master 2)

Ainsi, le retour en formation, s'il déstabilise certains acquis et amène à construire de nouveaux savoirs, peut devenir, à terme, un lieu de découverte des possibles, un moyen de 
Perez-Roux, T. (2016). Transitions professionnelles et transactions identitaires : expériences, épreuves, ouvertures. Revue Pensée plurielle, $n^{\circ} 41,81-93$.

développement professionnel et personnel, organisé autour d'un projet qui peu à peu se précise.

\section{L'épreuve de soi}

Dans les différentes études mobilisées, la transition suppose aussi une épreuve de soi. Le processus révèle quelque chose d'inattendu pour chacun, avec sa part objectivable (les progrès, les différents types de savoirs acquis, les compétences en actes) et sa part cachée, intime (les peurs, les déceptions, les obstacles insurmontables, etc.). Au-delà des difficultés, des moments de doute, notamment dans l'écriture du mémoire professionnel ou de recherche, reste le sentiment d'être allé au bout de l'aventure et d'en sortir grandi. Certains entretiens font état d'un réel sentiment de satisfaction : «finalement, j'ai fait mon petit bonhomme de chemin presque toute seule. J'ai eu de bons conseils, je les ai suivis. J'ai eu des échecs, ce qui m'a permis de redresser les épaules et de me dire : je vais pas m'arrêter maintenant. Et je vois mon évolution. La barque est lancée... elle ne va pas s'arrêter tout de suite... ». (Danielle, 32 ans, future enseignante en conduite routière). On perçoit par ailleurs une forme de légitimité accrue, pour soi-même et pour les autres : « ça m'a apporté une certaine assurance. Quand j'avance des choses, je n'ai plus peur, je sais qu'il y a un fond théorique derrière sur lequel je peux me baser pour avancer mes arguments. Ça me donne une légitimité et aussi des compétences bien précises. Parce qu'il y a des choses que je sais faire et que je ne soupçonnais pas que je pouvais faire » (Paco, 35 ans, commerce international, réorientation, en formation master 2)

Ainsi la transition renvoie à l'expérience de soi comme une épreuve solitaire et inédite, tout en étant irrémédiablement relationnelle et déterminée culturellement (Chiantaretto, 2008). Elle interroge le lien entre réalités extérieure et intérieure, ancrages dans le passé et projections dans le futur, relations de soi à soi et de soi à autrui.

En ce sens, les trois épreuves de l'altérité, des savoirs, de soi éclairent des dynamiques identitaires qui peuvent à présent être réinterrogées à partir des transactions.

\section{Des transactions identitaires à l'œuvre}

Comprendre les enjeux identitaires lors des transitions professionnelles nous amène à présent à revenir sur le modèle de la triple transaction.

$\mathrm{Au}$ plan biographique, la tension entre continuité et changement génère diverses formes de transactions. Le parcours antérieur -et les repères qui s'y sont construits- se trouve désormais requestionné, en prise avec la réalité du nouveau métier ou avec les exigences de l'université. Des transformations s'opèrent progressivement ou restent à opérer, en contexte de travail et de formation. Dans les études conduites, on ne repère pas d'éléments de rupture (réels ou symboliques) mais plutôt le sentiment, pour ces professionnels, de pouvoir s'adosser de façon plus explicite sur l'expérience acquise tout en envisageant l'avenir de façon plus ouverte : le changement opéré dans l'espace/temps de la transition semble amener de nouvelles ressources/stratégies pour penser, agir et en quelque sorte, se développer professionnellement. $\mathrm{Au}$ plan relationnel, on saisit l'importance des regards d'autrui sur la définition du soi professionnel. Le sentiment de légitimité se construit progressivement s'il est associé à des formes de reconnaissance qui font sens pour les acteurs. Pour Honneth (2000), sans la reconnaissance, l'individu ne peut se penser en sujet de sa propre vie et ne peut développer 
Perez-Roux, T. (2016). Transitions professionnelles et transactions identitaires : expériences, épreuves, ouvertures. Revue Pensée plurielle, $n^{\circ} 41,81-93$.

une relation positive à lui-même. La réalisation de soi, en tant que personne, dépend étroitement de cette reconnaissance mutuelle. Dans le cas de professionnels en transition, les formes de reconnaissance des pairs, des formateurs, des publics destinataires et, plus largement, de l'institution constituent un levier essentiel pour leur développement professionnel. Il s'agit en quelque sorte de «devenir capable - être reconnu - se (re)connaître » (Perez-Roux, 2012). Se reconnaître est ici entendu au sens de : se connaître comme en partie différent de celui que l'on était, que l'on projetait de devenir ou que l'on s'imaginait pouvoir être dans la réalité des situations d'enseignement ou de formation. Cette reconnaissance renvoie directement à l'estime de soi, et renforce des formes de légitimité pour soi-même et pour les autres essentielles pour investir sa (nouvelle) place/posture de professionnel.

Enfin la transaction intégrative (diversité-unité) se trouve fortement mise en jeu dans les moments de réflexivité, suscités par la formation. Les professionnels s'en saisissent peu à peu pour mettre en synergie réussites, ressources, idéaux, sans perdre de vue des préoccupations qui dépassent la sphère professionnelle : il s'agit de voir dans quelle mesure les différents registres d'activité et de pensée peuvent s'articuler et permettre de faire face aux exigences de la formation, du futur métier ou du métier revisité par l'expérience de la formation. On repère des formes de rééquilibrage entre sphère personnelle et professionnelle : « et puis, à côté de ça, je me suis achetée une petite maison. Peut-être que je vais apprendre à faire des petits trucs [travaux de réhabilitation], que je vais découvrir d'autres choses. Le master, ça m'a apporté ça. Aujourd'hui, j'ai envie de créativité ! ( Rosine).

Ainsi, le retour en formation et ses multiples effets mettent en lumière des reconfigurations du projet initial dans lesquelles se mêlent inextricablement le personnel et le professionnel. Les frontières sont poreuses tant au niveau de la genèse du passage effectif en formation qu'au niveau des potentialités qu'ouvre la formation et des nécessaires réactualisations en contexte. Le projet de soi prend alors une dimension intégrative essentielle : elle s'adosse à tel ou tel registre (professionnel, personnel, familial) qui permet le jeu des rééquilibrations. Comme le dit Marc à la fin de l'entretien, en guise de bilan : « J'aurais plein de choses à dire. Ça m'a fait énormément de bien de faire ce master et mon parcours, finalement, c'est le début de quelque chose, je le sens. Je me suis surtout trouvé moi. Personnellement j'ai énormément bougé des choses, enlevé des freins qui me servent dans ma vie de tous les jours, pas forcément dans ma vie professionnelle, mais dans ma façon d'aborder les choses. Un exemple : je vais être papa. Voilà, en avril. Je suis depuis très longtemps avec mon amie et il y avait un problème dans l'engagement. Donc ça a débloqué plein de choses. Voilà, je viens d'acheter un appartement. Tout arrive en même temps. Donc aujourd'hui ma vie est en train de bien bouger, de bien évoluer, en termes d'engagement, de maturité. Après, au niveau professionnel, il y a toujours ce petit truc qui me fait dire : mais vas-y, va faire ce dont tu as envie, donne toi cette chance ! La vie est courte, le temps passé au boulot non. Allez, oublie tes freins, mais bon... »

Derrière ces multiples remaniements, arrangements, désirs de réalisation, il s'agit pour chacun de retrouver un sentiment de cohérence, de se (re)donner une direction, une motivation, un sens.

\section{Conclusion}

Tenter de comprendre les transitions professionnelles et leurs effets sur les dynamiques identitaires reste un vaste chantier tant les enjeux sont complexes à appréhender. Un 
Perez-Roux, T. (2016). Transitions professionnelles et transactions identitaires : expériences, épreuves, ouvertures. Revue Pensée plurielle, $n^{\circ} 41,81-93$.

processus d'appropriation-distanciation-transformation apparaît de façon significative dans le cas de professionnels qui retournent en formation de façon volontaire. Au croisement de l'expérience passée et présente, il s'agit malgré tout de surmonter un certain nombre d'épreuves, de s'éprouver, pour faire la preuve et pour se prouver. Pour se trouver aussi, parfois.

\section{Bibliographie}

Alsène M., Gamache M., Lejeune M., 2002, Gestion des savoirs et gestion des compétences : une articulation possible mais limitée. Actes du 1er Colloque en Gestion des compétences et des connaissances, Nantes, pp. 27-31.

Balleux A., Perez-Roux T., 2013, «Autour des mots: Transitions professionnelles », Recherche et formation, $\mathrm{n}^{\circ} 74$, pp.101-114.

Boltanski L., Chiapello E., 1999, Le nouvel esprit du capitalisme, Paris, Gallimard.

Briançon M., 2012, L'Altérité enseignante. D'un penser sur l'autre à l'Autre de la pensée, Paris, Publibook.

Chiantaretto J-F., 2008, «L'altérité interne ou l'épreuve de soi. À propos de l'œuvre de Imre Kertész », Le Coq-héron 1/2008, n¹92, pp.18-25.

Cifali M., 1999, «Une altérité en acte : grandeurs et limites de l'accompagnement», in Chappaz G., Eds, Accompagnement et formation, Université de Provence et CDRP de Marseille, Marseille, pp.121-160.

Crozier M., Friedberg E., 1977, L'acteur et le système, Paris, Seuil.

Dubar C., 1992, «Formes identitaires et socialisation professionnelle », Revue Française de Sociologie, $\mathrm{n}^{\circ} 4$, pp.505-529.

Dubar C., 2000, La crise des identités. L'interprétation d'une mutation, Paris, PUF.

Dupuy R., Le Blanc A., 2001, «Enjeux axiologiques et activités de personnalisation dans les transitions professionnelles », Connexions, 2001/2, n 76, pp.61-79.

Honneth A., 2000, La lutte pour la reconnaissance, Paris, Cerf.

Kaddouri M., 2006, « Dynamiques identitaires et rapports à la formation »; in Barbier J-M., Bourgeois E., de Villers C., Kaddouri M, Eds., Constructions identitaires et mobilisation des sujets en formation, Paris, L'harmattan, pp.121-145.

Lanéelle X., Perez-Roux T., 2014, «Entrée dans le métier des enseignants et transition professionnelle : impact des contextes de professionnalisation et dynamiques d'acteurs », Revue de l'Orientation Scolaire et Professionnelle, $\mathrm{n}^{\circ} 43$ (4), pp.469-496.

Martuccelli D., 2006, Forgé par l'épreuve. L'individu dans la France contemporaine, Paris, Armand Colin.

Martuccelli, D., 2014, «La réversibilité des parcours, une utopie motrice », Sociologies pratiques, $\mathrm{n}^{\circ} 28$, pp.11-18.

Mazade O., Hinault A-C., 2014, «Avant-propos », Les identités professionnelles à l'épreuve des transitions, Sociologies pratiques, n²8, pp.3-8.

Megemont J-L., Baubion-Broye A., 2001, « Dynamiques identitaires et représentations de soi dans une phase de transition professionnelle et personnelle », Connexions, $\mathrm{n}^{\circ}$ 76, pp.15-28.

Perez-Roux T., 2010, «Processus de professionnalisation et dynamiques identitaires : deux études de cas chez les enseignants de lycée professionnel en France », Nouveaux cahiers de la recherche en éducation, Québec, $\mathrm{n}^{\circ} 13(1)$, pp.83-101.

Perez-Roux T., 2011, "Changer de métier pour devenir enseignant: transitions professionnelles et dynamiques identitaires », Recherches en éducation, ${ }^{\circ} 11$, pp.39-54. 
Perez-Roux, T. (2016). Transitions professionnelles et transactions identitaires : expériences, épreuves, ouvertures. Revue Pensée plurielle, n 41, 81-93.

Perez-Roux T., 2012, «Construction identitaire des enseignants débutants: quelle reconnaissance d'autrui pour se (re)connaitre en tant que professionnel ?», Recherches et éducations, $\mathrm{n}^{\circ}$ 7, pp.69-84.

Perez-Roux T., Balleux A., 2014, Mutations dans l'enseignement et la formation : brouillages identitaires et stratégies d'acteurs, Paris, L'Harmattan.

Rose J., 2009, «La place de la formation dans les politiques de transition professionnelle », in Barbier J-M., Bourgeois E., Chapelle G., Ruano-Borbalan J-C, Eds, Encyclopédie de la formation, Paris, PUF, pp.353-383.

Tardif M., Lessard C., 1999, Le travail enseignant au quotidien. Expériences, interactions humaines et dilemmes professionnels, Bruxelles, De Boeck.

T. Perez-Roux

Université P. Valéry, UFR6

Route de Mende

34199 Montpellier cedex 5

Tel bureau : 0467142539

Portable : 0660783949 